Man verdampft die Zinklösung in einer grösseren Platinschale auf dem Wasserbade zur Trockne (ein Umrühren ist am Schluss nicht erforderlich), erhitzt die rückständige Salzmasse ungefähr eine Stunde im Luftbade auf 125 bis $150^{\circ} \mathrm{C}$. und verjagt dann die Ammonsalze durch stärkeres Erhitzen auf einer Asbestschale über der Gasflamme. Die an den Seitenwänden haften bleibenden Salzmassen müssen zeitweilig mittelst eines starken Platindrahtes auf den Boden der. Schale herabgestossen werden. Ein Verjagen der Ammonsalze, unmittelbar über der Flamme, ist aber nicht zulässig, da hierbei Verluste an Zink nicht ausgeschlossen sind. Der nicht flüchtige Rückstand wird num in heissem Wasser und einigen Tropfen Salzsäure gelöst und diese Lösung nach Filtration in einer echten Porzellanschale kochend mit Natriumcarbonat gefällt.

Die Verfasser führten quantitative Trennungsversuche mit reinem Mangansulfat und Zinkvitriol aus und erzielten hierbei günstige Resultate.

Ein Vorzug der Methode besteht darin, dass sich der ergebende Manganniederschlag ganz besonders leicht filtriren und rasch auswaschen lässt, aus welchem Grunde eine Manganbestimmung neben Zink bequem und genau in einem Zeitraume von zwei Stunden ausführbar ist.

Die Verfasser sind gegenwärtig mit Versuchen beschäftigt, bei welchen dieselben das bei der beschriebenen Methode zur Anwendung kommende Princip auch zu der Trennung des Mangans von anderen Metallen zu benutzen suchen.

\title{
Zur Bestimmung des Phosphors in Roheisen, Stahl und Eisen-
} erzen. In den Vereinigten Staaten von Nordamerika ist eine von F. A. Emmerton ${ }^{1}$ ) vorgeschlagene Nethode in Gebrauch, nach welcher der Phosphor im Eisen auf indirectem Wege in der Weise bestimmt wird, dass man in dem Sonnenschein'schen Niederschlag die Menge der Molybdänsäure feststellt. Zur Bestimmung der Molybdänsäure wird der Niederschlag in Ammoniak gelöst, die Molybdänsäure mit Zink und Schwefelsäure reducirt und die reducirten Oxyde mit Kaliumpermanganatlösung titrirt, wobei sich wieder Molybdänsäure bildet. Da in dem gelben Molybdänniederschlage sowohl die Menge der Molybdänsäure, als auch diejenige des Phosphors genau bekannt ist, lässt sich aus der ermittelten Molybdänsäure der vorhandene Phosphor leicht berechnen.

1) Andrew Alexander Blair, The Chemical Analysis of Iron, second edition, S. 95; vom Verfasser eingesandt. 
Die Molybdänsäure wird bei der Behandlung mit Zink und Schwefelsäure nicht $\mathrm{zu} \mathrm{Mo}_{2} \mathrm{O}_{3}$ reducirt, sondern zu einem Gemenge von Oxyden, welches der Formel $\mathrm{Mo}_{12} \mathrm{O}_{19}$ entspricht. Die Einwirkung des Kaliumpermanganats auf die bei der Reduction gebildeten Oxyde wird durch nachstehende Gleichung veranschaulicht:

$5 \mathrm{Mo}_{12} \mathrm{O}_{19}+17\left(\mathrm{~K}_{2} \mathrm{OMn}_{2} \mathrm{O}_{7}\right)=60 \mathrm{MoO}_{3}+17 \mathrm{~K}_{2} \mathrm{O}+34 \mathrm{MnO}$.

17 Molecüle Permanganat entsprechen demnach 60 Molecülen Molybdänsäure, und 1 Molecül Permanganat, welches 560 Theile Eisen aus dem Oxydulzustande in Oxyd überzuführen vermag, bildet aus $\mathrm{Mo}_{12} \mathrm{O}_{19}$ 508,23 Theile Molybdänsäure; bei einer auf Eisen gesteliten Permanganatlösung entsprechen daher 100 Theile Eisen 90,76 Theilen Molybdänsäure. Um bequemes Rechnen zu ermöglichen, bereitet man zweckmässig eine Permanganatlösung, von welcher $1 \mathrm{ec}$ 0,0001 $g$ Phosphor entspricht; von dieser Lösung entspricht $1 c c 0,006141 \mathrm{~g}$ Eisen und, $90,76 \%$ von dieser Zahl, 0,005574g Molybdänsäure. Da in dem phosphormolybdänsauren Ammonniederschlag der Gehalt an Phosphor $1,794 \%$ der vorhandenen Molybdänsäure beträgt, ${ }^{1}$ ) sind demnach für 1 cc der Permanganatlösung 0,0001 $g$ Phosphor in Rechnung zu stellen. Bei Ausführung einer Bestimmung verfăhrt man nach Emmerton in folgender Weise:

Man löst $5 \mathrm{~g}$ Stahl in einer Porzellanschale von etwa $15 \mathrm{~cm}$ Durchmesser in $75 c c$ Salpetersäure von 1,2 specifischem Gewicht. Die Schale bedeckt man hierbei mit einem grossen Uhrglase, welches den Rand der Schale etwa $1 \mathrm{~cm}$ überragt. Die Lösung kocht man rasch auf einer heissen Eisenplatte ein und erhitzt, nachdem der Rückstand trocken erscheint, noch etwa 30 Minuten auf der. Platte. Man lässt erkalten, fügt $40 c c$ starke Salzsäure hinzu, legt das Uhrglas dicht auf die Schale und erhitzt einige Minuten gelinde, bis das an den Deckel gespritzte Eisenoxyd erweicht ist. Hierauf kocht man die Flüssigkeit bis auf $15 \mathrm{cc}$ ein. Dieses Einkochen verlangt besondere Aufmerksamkeit, da die Lösung stark concentrirt werden soll, ohne dass dabei Eisenchlorid an. den Wandungen der Schale eintrocknet. Man lässt die Schale etwas abkühlen, nimmt das Chrglas weg und spült dasselbe mit 40 ce starker Salpetersäure in die Schale ab. Die letztere wird nun mit einem Uhrglase bedeckt, dessen concave Seite nach Innen gerichtet ist, und welches im Durchmesser etwa $2 \mathrm{~cm}$ kleiner wie die Schale ist, so dass bei dem

1) In dem Niederschlage sind auf $24 \mathrm{M}_{0} \mathrm{O}_{3} 1 \mathrm{P}_{2} \mathrm{O}_{5}$ enthalten. 
jetzt erfolgenden Einkochen die Flüssigkeit, welche sich an der inneren Seite des Uhrglases condensirt, an den Wandungen der Schale zurückfliesst, nicht aber in die Mitte derselben abtropft. Es wird hierdurch verhindert, dass sich an dem Rande der Flüssigkeit eine Kruste bildet. Ist die Flüssigkeit in der so bedeckten Schale auf $15 \mathrm{cc}$ eingekocht, so nimmt man dieselbe von der Platte und sucht durch Bewegen der Schale etwa gebildete Ausscheidungen wieder in Lösung zu bringen. Auf diese Weise gelingt es bei einiger. Uebung eine sehr concentrirte und vollständig klare Lösung zu erhalten, aus welcher alle Salzsäure ausgetrieben ist. Die etwas erkaltete Lösung verdünnt man mit Wasser auf etwa $40 c c$ und spült dieselbe in einen $400 c c$ fassenden Kolben, wobei sich etwa $75 \mathrm{cc}$ Flüssigkeit ergeben. Man versetzt nun vorsichtig und unter öftẹrem Umschätteln mit starkem Ammoniak, bis die Flüssigkeit zu einer steifen Gallerte gesteht; alsdann fügt man unter Umschütteln noch einige Cubikcentimeter Ammoniak mehr zu, so dass die Flüssigkeit einen starken Geruch nach Ammoniak zeigt. Hierauf wird allmählich unter beständigem Umschütteln mit starker Salpetersäure versetzt, bis die Flüssigkeit dünner wird. Ist der Niederschlag gelöst, und zeigt die Lösung eine sehr dunkele Farbe, so gibt man noch ein wenig mehr Salpetersäure zu. Das Volumen der alsdann Bernsteinfarbe zeigenden Flüssigkeit beträgt nun $150-300 \mathrm{cc}$, gewöhnlich etwa $250 \mathrm{cc}$.

Man bestimmt jetzt die Temperatur der so erhaltenen Lösung und bringt dieselbe auf $85^{\circ} \mathrm{C}$, indem man die Lösung entweder vorsichtig über einer Flamme erhitzt, oder in kaltem Wasser bis zu dieser Temperatur abkühlt. Sobald man die Lösung auf die Temperatur von $85^{\circ} \mathrm{C}$. gebracht hat, versetzt man dieselbe in einem Kolben auf einmal mit 40 cc Molybdänlösung ${ }^{1}$ ). Der Kolben wird nun mit einem Gummistopfen verschlossen, in ein dickes Tuch gewickelt und 5 Minuten lang heftig geschüttelt. Nach Ablauf dieser Zeit ist die Fällung eine vollständige.

Man sammelt nun den Niederschlag auf einem Filter unter Anwendung der Saugpumpe und wäscht denselben mit verdünnter Salpetersäure aus, welche man durch Verdünmen der gewöhnlichen Säure mit der 50 fachen Menge Wasser herstellt. Sollte hierbei etwas von dem

1) Die Molybdänlösung bereitet man durch Auflösen von $100 \mathrm{~g}$ Molybdänsäure in einer Mischung von $300 \mathrm{cc}$ starkem Ammoniak und $100 \mathrm{cc}$ Wasser; diese Lösung giesst man in $1250 \mathrm{cc}$ Salpetersäure von 1,2 specifischem Gewicht. 
Niederschlage an den Wandungen des Kolbens haften bleiben, so wird der letztere mit dem Ammoniak ausgespült, welches man zum Lösen des Molybdänniederschlags verwendet. Letzteres bewirkt man in der Weise, dass man den Trichter mit dem noch feuchten Niederschlag auf einen zweiten $500 \mathrm{cc}$ fassenden Kolben, in welchem sich, annäherend gewogen, $10 \mathrm{~g}$ granulirtes Zink befinden, setzt, die Spitze des Filters durchstösst und den Niederschlag mit verdünntem Ammoniak $(1: 4)$ in den Kolben spült. Bei dieser Operation sollen nicht mehr wie $30 c c$ Ammoniak verbraucht werden. Man giesst dann in den Kolben $80 \mathrm{cc}$ heisse verdünnte Schwefelsäure $(1: 4)$, hängt in den Hals desselben einen kleinen Trichter und erhitzt sofort auf einer Eisenplatte, bis das Zink sich rasch zu lösen beginnt. Nachdem man hierauf das Erhitzen vorsichtig 10 Minuten lang fortgesetzt und eine vollständige Reduction der Molybdänsäure erreicht hat, giesst man die Lösung, um dieselbe von dem ungelösten Zink zu trennen, durch ein grosses Faltenfilter. Den Kolben spült man mit kaltem Wasser nach und füllt das Filter, sobald die ersten Waschwasser durchgelaufen sind, noch einmal mit kaltem Wasser an. Bei dieser Filtration wird die Flüssigkeit nur kurze Zeit der Einwirkung der Luft ausgesetzt, und, da das ungelöste Zink mit auf das Filter gebracht wird, setzt dasselbe seine reducirende Wirkung fort, bis die Flüssigkeit abgelaufen ist.

Das 400 bis $500 \mathrm{cc}$ betragende Filtrat ist jetzt für die Titration mit Permanganatlösung fertig; von der letzteren lässt man so lange zufliessen, bis die Flüssigkeit eben farblos geworden ist.

Während der Reduction der Molybdänsäure nimmt die Flüssigkeit aufeinander folgend verschiedene Farben an; die bei beginnender Reduction blassroth erscheinende Flüssigkeit wird schwach- und schliesslich dunkel-olivgrün. Die Stärke der Endfarbe hängt von der Menge der reducirten Molybdänsäure ab. Sobald die reducirte Flüssigkeit auf das Filter kommt, verschwindet die grüne Farbe derselben and geht in Portweinfarbe über, doch scheint diese Farbenänderung nicht durch eine merklìche Oxydation hervorgerufen zu sein, da die Oxydation der reducirten Flüssigkeit nur langsam erfolgt.

Bei der Titration wird die Farbe der Flüssigkeit heller und verschwindet zuletzt; man erhält eine vollständig farblose Flüssigkeit, in welcher ein weiterer Tropfen Permanganatlösung eine hellrothe Färbung hervorruft. 
Bei Roheisen löst man $5 g$ und behandelt die salpetersaure Lösung wie oben. Die sich ergebende salzsaure Lösung spült man sammt dem unlöslichen Rückstand in einen $100 \mathrm{cc}$ fassenden Messkolben, füllt bis zur Marke auf, mischt und filtrirt durch ein trockenes Filter in einen trockenen Kolben unter Anwendung der Saugpumpe. Von dem Filtrate werden $80 \mathrm{cc}$, entsprechend $4 \mathrm{~g}$ Eisen, weiter verarbeitet.

Bei Anwendung der Methode auf Eisenerze löst man $10 \mathrm{~g}$ in Salzsäure, verdampft die Lösung zur Trockne und nimmt den Rückstand wieder mit Salzsäure auf. Dann wird auf ein kleines Volumen abgedampft und die Salzsäure durch Einkochen mit $40 \mathrm{cc}$ starker Salpetersäure ausgetrieben. Bevor man hierauf die weitere Behandlung vornimmt, filtrirt man den ungelöst bleibenden Rückstand ab. Zum Schütteln der Flüssigkeit bei der Abscheidung des Molybdänniederschlags ist die Anwendung eines Schüttelapparates anzurathen, namentlich wenn zahlreiche Bestimmungen auszuführen sind. Emmerton beschreibt einen für den genannten Zweck besonders geeigneten Apparat.

Gegen diese Methode macht $\mathrm{Clemens} \mathrm{J}_{0} \mathrm{es}^{1}$ ) verschiedene Bedenken geltend. Derselbe führt mehrere Versuche nebst kritischen Bemerkungen an, welche er der Mittheilung von A. L. Colby verdankt. Aus den mitgetheilten Versuchen geht hervor, dass die Anwendung von verdünnter Salpetersäure oder einer angesäuerten Lösung von salpetersaurem Ammon zum Auswaschen des Molybdänniederschlags von nachtheiligem Einfluss ist, da die Gegenwart von Salpetersäure in der zuletzt erhaltenen zu reducirenden Lösung den Verbrauch an Permanganatlösung wesentlich erhöht. Die verdünnte Salpetersäure lässt sich nun nach den Versuchen von Colby zweckmässig durch verdünnte Schwefelsäure (1:50) ersetzen; der Molybdänniederschlag wird von dieser Säure selbst. bei einer Verdünnung von nur 1:5 nicht merklich gelöst. Noch empfehlenswerther zum Auswaschen des Niederschlags erscheint eine Lösung von schwefelsaurem Ammon, indem die Versuche bei Verwendung dieser Lösung bessere Uebereinstimmung zeigten, als bei dem Auswaschen mit verdünnter Schwefelsäure. Bei sämmtlichen Versuchen brachte Colby weiter für das in dem Zink enthaltene Eisen eine Correctur an, welche durch einen besonderen Versuch festgestellt wurde.

P. W. Shimer hat gleichfalls zum Auswaschen des Molybdänniederschlags verdünnte Schwefelsäure und schwefelsaures Ammon vor-

1) Transactions of the American Institute of mining engineers, Washington meeting, February 1890; vom Verfasser eingesandt. 
geschlagen, und ausserdem die Anwendung von Kaliumpermanganat als Oxydationsmittel bei dem Lösen des Eisens empfohlen. Von diesem Vorschlag hat bereits Thomas M. Drown Gebrauch gemacht, indem derselbe Roheisen oder Stahl in Salpetersäure von 1,135 specifischem Gewicht unter gleichzeitiger ALwendung von Kaliumpermanganat löste, bei welcher Behandlung der vorbandene Phosphor sicher in Orthophosphorsäure übergeführt wurde.

Jones hat die Methode weiter modificirt und schlägt vor in folgender Weise zu verfahren:

Man löst Roheisen oder Stahl in Salpetersäure von 1,135 specifischem Gewicht, erhält nach Beendigung der Reaction die Lösung eine Minute lang im Kochen und fügt alsdann vorsichtig eine Lösung von Kaliumpermanganat hinzu, bis sich Mangandioxyd abscheidet. Das letztere bringt man durch Zufügen von einigen kleinen Krystallen schwefelsaurem Eisenoxydul ${ }^{1}$ ) wieder in Lösung. Man filtrirt in einen $500 \mathrm{cc}$ fassenden Kolben und fügt Ammoniak von 0,90 specifischem Gewicht zu bis zur fast vollständigen Neutralisation. Die Lösung wird nun erhitzt und mit wenigen Tropfen Permanganatlösung versetzt. Etwa sich abscheidendes Mangandioxyd wird durch schwefelsaures Eisenoxydul wieder gelöst und die Lösung bei $85^{\circ} \mathrm{C}$. mit $75 \mathrm{cc}$ Molybdänlösung versetzt. Nach fünf Minuten langem Schütteln wird der Niederschlag unter Anwendung der Saugpumpe filtrirt und mit einer Lösung von schwefelsaurem. Ammon ausgewaschen. Den Niederschlag löst man auf dem Filter in Ammoniak von 0,96 specifischem Gewicht, ohne das Filter zu durchstossen, und wascht dasselbe ein- oder zweimal unter Absaugen mit Wasser aus. Man giesst die ammoniakalische Lösung aus dem Kolben. in ein kleines Becherglas, spült dreimal mit Wasser nach und filtrirt die Lösung wieder durch das ursprüngliche Filter in den Kolben zurück, wobei manl jetzt das Filter vollständig mit Wasser auswäscht und die Lösung schliesslich auf $300 c c$ verdünnt.

Zum Reduciren der Molybdänsäure benutzt Jones den von ihm vorgeschlagenen Reductionsapparat. ${ }^{2}$ ) Nachdem man das Zink in dem Apparate in der vorgeschriebenen Weise mit verdünnter Schwefelsäure gereinigt hat und der Apparat so zur Reduction vorbereitet ist, wird

1) Das zu verwendende schwefelsaure Eisenoxydul muss selbstrerständlich frei von Phosphorsäure sein; da dasselbe nur schwer ganz rein zu erhalten ist, muss eventuell eine entsprechende Correctur angebracht werden.

2) Diese Zeitschrift 29, 597. 
die ammoniakalische Lösung des Molybdänniederschlags mit 30 bis $50 \mathrm{cc}$ Schwefelsäure von 1,32 specifischem Gewicht versetzt und die sich ergebende Flüssigkeit nun unter starkem Saugen durch den Reductionsapparat filtrirt. Das Nachwaschen des Apparats geschieht wie bei der Reduction von Eisenoxydsalzen, und die Titration der gesammten Lösung wird alsdann in bekannter Weise vorgenommen. Die durch die Verunreinigungen des Zinks bedingte Correctur ist, wie früher beschrieben, festzustellen and in Abzug zu bringen.

Bei Anwendung der Methode auf Eisenerze wird das Erz in Salzsäure von 1,12 specifischem Gewicht gelöst und der unlösliche Rückstand ahfiltrirt. Das Filtrat wird mit Salpetersäure von 1,20 specifischem Gewicht verdampft, der Rückstand mit kohlensaurem Natron geschmolzen ${ }^{1}$ ), die Schmelze in. Wasser und verdünnter Schwefelsäure gelöst und diese Lösung mit der salpetersauren Lösung vereinigt. Eine Abscheidung der Kieselsäure aus der Lösung der Schmelze erscheint unnöthig, da Kieselsäure die Bestimmungen nach dieser Methode nicht beeinflusst. Die vereinigten Lösungen werden in einem Kolben mit Kaliumpermanganatlösung oxydirt und alsdann in der oben beschriebenen Weise weiter behandelt.

Bei Roheisen oder Stahl, welche Arsen oder Titan enthalten, ist die Methode nicht direct anwendbar. Das Arsen muss stets abgeschieden werden und erschwert die Anwendung der Methode wesentlich, doch ist dies bei jeder anderen Methode mehr oder weniger auch der Fall.

Ebenso beeinflusst das Vorhandensein von Titan die Bestimmungen nach dieser Methode. Arbeitet man nach der Vorschrift von Emmerton und verdampft die erste Lösung zur Trockne, so wird der durch das Titan verursachte Fehler zwar verringert, doch sind bei grösseren Mengen von Titan die Resultate auch dann ungenau.

Jones theilt zahlreiche Versuche mit, welche sehr günstige Resultate ergeben haben, indem dieselben sowohl unter einander, als auch mit nach anderen Methoden erhaltenen Resultaten sehr gute Uebereinstimmung zeigen. Nach dieser Methode lässt sich die Bestimmung des Phosphors in einem Eisen, welches weder Arsen, noch Titan enthält, ohne Schwierigkeit in nicht ganz einer Stunde ausführen; ebenso erfordert die Bestimmung in Eisenerzen nicht viel mehr Zeit.

1) Das Schmelzen des Rückstandes verursacht keinen besonderen Zeitrerlust und ist als vorsichtig unter allen Umständen anzurathen. 